\title{
The interferometric view of Herbig Ae/Be stars
}

\author{
Stefan Kraus
}

\begin{abstract}
In this contribution I review how optical interferometry has contributed to shaping our understanding of the class of Herbig Ae/Be stars and of their associated circumstellar disks. I discuss the evidence for an optically-thin cavity in the inner few astronomical units (au) and a "puffed-up rim" near the dust sublimation radius and how these observations helped to establish the current generation of irradiated disk models. Multi-wavelength interferometric observations also revealed systems with clear signatures of grain growth and dynamically-cleared disk gaps, tracing important stages of disk evolution and of ongoing planet formation. I discuss the new opportunities provided by spectro-interferometry, which enables detailed studies on the gas distribution and velocity field on sub-au scales, resulting in constrains on the accretion properties of the system and the disk excitation structure. Finally, I outline some of the open questions and loose ends in current interferometric studies, and how these might point towards new disk physics.
\end{abstract}

Keywords Herbig Ae/Be stars; protoplanetary disks; accretion; star formation; planet formation

\section{Introduction}

While it is well established that dust grains are responsible for the infrared excess emission in the intermediate-mass Herbig $\mathrm{Ae} / \mathrm{Be}(\mathrm{HAeBe})$ stars, the three-dimensional distribution of this material is still being debated. Until recently, the spatial scales of the inner circumstellar environment (a few astronomical units) were not accessible to optical and infrared imaging observations, and conclusions drawn on the spatial

Stefan Kraus

School of Physics, University of Exeter, Stocker Road, Exeter, EX4 4QL, UK

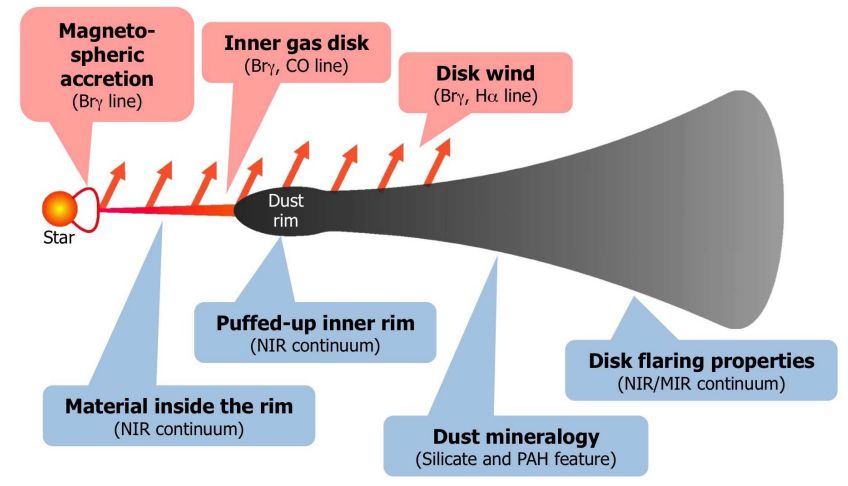

Fig. 1 Sketch of the disk components that have been studied with NIR and MIR long-baseline interferometry, both in spectral lines (top) and in the dust continuum emission (bottom).

distribution of the circumstellar material were, in most cases, entirely based on the modeling of the spectral energy distribution (SED). However, as demonstrated by Thamm et al. (1994) and others, these SED model fits are highly ambiguous, and only the combination of SED modeling with high-resolution imaging can provide crucial constraints on the real geometry of the circumstellar matter.

Interferometry at visual and infrared wavelengths allows us, for the first time, to study the distribution of the circumstellar gas and dust in HAeBe stars directly, probing fundamental aspects of our paradigm of disk structure and physics (Fig. 1). In this paper I will review how these observations have started to guide our understanding of this intruiging object class. Near-infrared (NIR) and mid-infrared (MIR) interferometry provided exciting new constraints on the disk structure near the dust-sublimation rim (Sect. 2) and in the extended disk at intermediate disk radii out to a few tens of astronomical units (Sect. 3). Interferom- 
etry has also allows us to detect and characterise gaps and disk asymmetries (Sect. 4), providing potentially an important link to planet formation theories. Another promising research field is to use interferometry with high spectral dispersion in order to constrain the dust mineralogy (Sect. 5) and gas kinematics in different diagnostic spectral lines (Sect. 6). Finally, I will discuss interferometric studies related to the multiplicity of Herbig stars (Sect. 7), before concluding in section 8 .

Throughout the paper I will focus on the (already rather extensive) work that has been done on HAeBe stars, while results on T Tauri stars, FU Orionis stars, and supergiant-B[e] stars are out of the scope of this paper.

\section{Disk structure near the dust sublimation radius}

Already the first infrared interferometric observations on Herbig stars with the IOTA (Millan-Gabet et al. 1999, 2001) and PTI (Akeson et al. 2000) interferometer were able to measure the characteristic sizes of a large number of Herbig disks at NIR wavelengths $(H$ and $K$-band). Combining the light of just two telescopes, these early observations were not able to constrain the detailed brightness distribution, but derived the characteristic size of the emitting region by fitting simple geometric models (e.g. rings or Gaussians) to the measured visibility amplitudes. In general, the measured disk sizes were larger than predicted by the viscous accretion disk models that were commonly used at that time and that extended inwards to the stellar surface (e.g. Hillenbrand et al. 1992). These observational results triggered further theoretical work on irradiated disk models with a dust-free central cavity and a frontally-heated "puffed-up" inner disk rim (Natta et al. 2001; Dullemond et al. 2001). The puffed-up rim might also cast a shadow on the outer disk regions, with strong implications on the dust temperature in the more extended disk.

Monnier and Millan-Gabet (2002) collected NIR disk size measurements from different surveys and plotted the measured disk sizes as function of stellar luminosity. The resulting "size-luminosity diagram" (Fig. 2a) shows that the disk sizes scale roughly with the square-root of the stellar luminosity, providing support to the idea that the NIR emission is dominated by the emission from the dust sublimation rim.

One interesting result from these studies is that lowmass objects $\left(\lesssim 20 L_{\odot}\right)$ tend to be more extended than the predicted sublimation radius (Fig. 2a). Pinte et al. (2008) showed that this effect can likely be explained by (a) Size-luminosity relation:

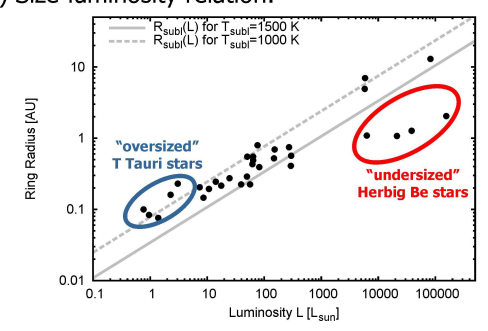

(b) Temperature gradient measurements:

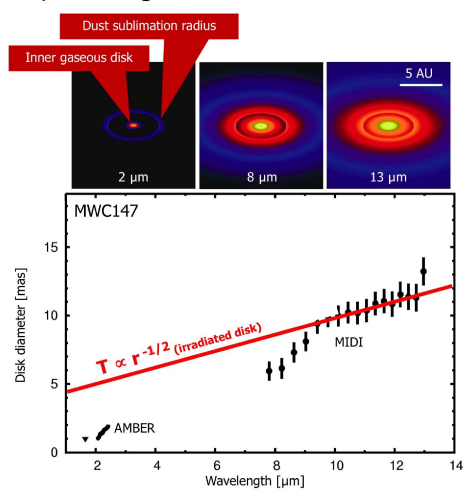

(c) Interferometry at baselines $>200 \mathrm{~m}$ :

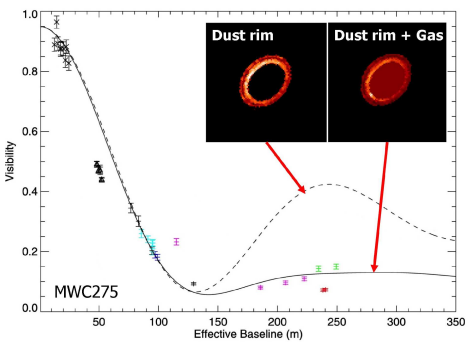

(d) Imaging:

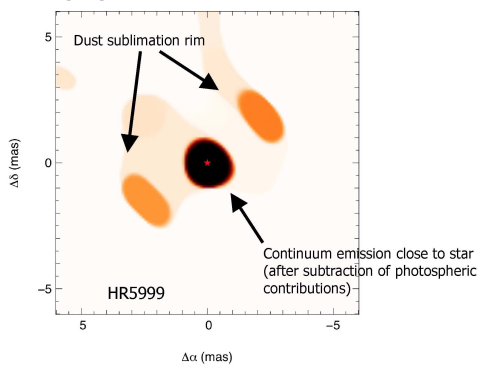

Fig. 2 Some of the evidence indicating large NIR continuum contributions from inside of the dust sublimation rim: (a) The size-luminosity diagram reveal a population of "undersized" Herbig Be stars, with sizes smaller than the expected dust-sublimation radius (Monnier et al. 2005); (b) Combined NIR+MIR interferometry on MWC147 constrainted the temperature distribution in the disk, indicating the presence of a very hot emission component located inside of the dust rim (Kraus et al. 2008a); (c) CHARA interferometry at baselines $\gtrsim 200 \mathrm{~m}$ has shown that the visibility profile of MWC275 does not exhibit a pronounced $2^{\text {nd }}$ lobe, suggesting a smooth intensity profile instead of a sharp opacity change near the dust rim (Tannirkulam et al. 2008); (d) VLTI imaging of HR5999 found significant NIR emission from close to the star, even after the photospheric contributions were subtracted (Kluska et al. 2014). 
the fact that the fractional contributions from scattered light should increase for stars of later spectral type. Cooler stars radiate a larger fraction of their bolometric luminosity at NIR wavelengths and the direct thermal emission therefore decreases faster with radius than the scattered light contributions.

High-luminosity Herbig Be stars, on the other hand, tend to be "undersized" compared to the expected dust sublimation radius (Fig. 2a). Several effects have been proposed to explain this effect:

(a) Gas absorption in the inner disk could shield the dust partially from the stellar radiation and thus allow dust to exist closer to the star (Monnier and Millan-Gabet 2002; Muzerolle et al. 2004).

(b) Gas emission contributions from inside of the dust sublimation rim could contribute to the NIR excess and make the object appear more compact (Eisner et al. 2004; Kraus et al. 2009b).

(c) Highly refractory dust grain species with sublimation temperatures far beyond $2000 \mathrm{~K}$ might be able to survive well inside of the conventional dust sublimation zone for Silicate or Carbonaceous dust (Benisty et al. 2010a).

Each of these effects could contribute to the measured small disk sizes, but further efforts both in modelling and observation will be needed in order to identify the dominant effect. Modelling-related open questions are whether the gas opacities and the scale height of the gas disk are sufficiently high in order to provide efficient shielding (scenario a) and whether the gas would result in molecular emission features that are not observed in the SED (scenario b). For scenario c, it is not clear yet which dust species could survive at the required high temperatures.

Following the first generation of interferometry surveys outlined above, more detailed studies were conducted and aimed to constrain both the radial intensity profile and the disk inclination angle for individual objects. Eisner et al. (2004) measured the inclination for about a dozen of HAeBe stars. Kreplin et al. (2013) addressed the origin of the UX Orionis-type brightness variability that is observed in some Herbig stars. It has been proposed that such photometric variability could be caused by obscurations of the central star in an almost edge-on disk. The team observed the Herbig Ae star KKOphiuchus and measured an inclination angle of $70^{\circ}$, which is consistent with the prediction that UX Ori objects are seen under large inclination angles and that the observed variability might be caused by orbiting clouds passing through the line of sight.
During the last decade, new observational capabilities have become available that allow us now to step beyond size and elongation measurements and to obtain more detailed information about the disk structure near the dust sublimation zone. The most significant advancements in this respect came from:

Phase closure interferometry: Combining 3 or more telescopes, IOTA, VLTI, and CHARA have enabled phase closure observations, which provide a good measure for asymmetries in the brightness distribution. The first IOTA closure phases on YSOs (with relatively short baseline lengths) revealed small phase signals that were in most cases consistent with a centrosymmetric brightness distribution (Millan-Gabet et al. 2006; Monnier et al. 2006). This result was interpreted as evidence that the dust sublimation rim is less sharp than predicted by rim models with a perfectly vertical truncated disk rim (Dullemond et al. 2001). More detailed studies with longer baselines were conducted on individual objects. For instance, Kraus et al. (2009a) measured significant non-zero closure phase signals on R Coronae Australis. The visibility and closure phase signals also varied systematically with baseline position angle, which could be modelled with a curved rim model that takes the gas pressure-dependence of the dust sublimation temperature into account (Isella and Natta 2005).

Phase closure observations with good $u v$-coverage enable also the reconstruction of model-independent interferometric images. First YSO imaging results have been presented on IRAS13481-6124 (Kraus et al. 2010), MWC275 (=HD163296, Renard et al. 2010), and HR5999 (Benisty et al. 2010a; Kluska et al. 2014). The images generally confirm the modelling results that the emission is more compact than the expected location of the dust sublimation rim for commonly assumed Silicate sublimation temperatures of $\sim 1500 \mathrm{~K}$. Also, the image on HR 5999 shows larger flux contributions than just the photospheric flux inside of the dust sublimation rim. Kluska et al. (2014) applied a method that subtracts the chromatic contributions from the star and thereby allows to combine data taken over a wider wavelength range. Their study determines that $60 \%$ of the $K$-band flux in HR5999 origins from inside of the dust rim, far exceeding the $22 \%$ that have been determined with SED modelling (Fig. 2d).

Multi-wavelength interferometry: With the advent of spectrally dispersed interferometry (spectrointerferometry), it has become possible to constrain also the temperature distribution of the emitting material. First constraints on the temperature distribution in HAeBe stars were obtained by fitting the visibility slope within the $K$-band (Eisner et al. 2007). Using a disk model with temperature power law $(T(r) \propto$ 
$\left.T_{\text {in }}\left(R / R_{\text {in }}\right)^{-\alpha}\right)$, they found $\alpha$ values between 0.4 and 1.0, where early-type stars show a slightly steeper temperature gradient. In order to address the question of the "undersized" Herbig Be stars, Kraus et al. (2008a) studied the Herbig Be star MWC147 with combined NIR+MIR interferometry. MIDI and AMBER observations revealed the presence of two temperature components that could be modelled with a hot, viscously heated accretion disk component inside of the dust sublimation radius plus an irradiated disk (Fig. 2b). These results add support to the idea that the surprisingly compact emission in some Herbig Be stars might be due to optically thick gas located inside of the dust sublimation radius.

Interferometry on longer baselines: The first generation of interferometric measurements on YSOs were conducted with relatively short baselines $(\lesssim$ $100 \mathrm{~m}$ ) and probed only the first lobe of the visibility function. A major advancement can be expected from observations at longer baselines, for instance with the CHARA array that offers baselines up to $330 \mathrm{~m}$. Observations on the Herbig stars MWC275 and AB Aurigae have been presented (Tannirkulam et al. 2008) and revealed a very low fringe contrast on the longest baselines (Fig. 2c), indicating that the radial emission profile at the dust sublimation rim is not as sharp as predicted by dust rim models.

All of the aforementioned results reinforce the finding that the current disk models still lack continuum emission originating from inside of the dust sublimation radius, which might be related either to gas located inside of the dust rim, unconventional dust grain species, or another physical effect that has not been identified yet. Recently, new models of dusty disk winds (Bans and Königl 2012) and magnetically-support disk atmospheres (Turner et al. 2014) have been presented. In these models, dust is lifted by magnetic forces out of the disk plane and might add opacity close to star for systems seen under inclination. It will be essential to test such models with observations at very high spatial frequencies.

\section{Disk structure in the extended disk}

The NIR visibility profiles of many HAeBe stars reveal the presence of very extended NIR emission, as indicated by a drop of the visibility function at very short spatial frequencies. This extended emission can contribute up to $\sim 30 \%$ of the total $H$ and $K$-band flux in some systems (e.g. Kraus et al. 2009a) and is typically distributed over a few hundred milliarcseconds to a few arcseconds (Leinert et al. 2001). The physical origin of the emission is still debated, but it might correspond to scattered light from the outer disk (Pinte et al. 2008), from an halo in which the disk is embedded, or the walls of an outflow cone. Making further progress on this field will require systematic studies on a larger sample of disks, with the goal to reveal correlations with the stellar mass, dust properties, the global disk geometry (e.g. flared versus self-shadowed) or evolutionary stage of the system.

Another efficient method for tracing the disk regions beyond the dust sublimation rim is mid-infrared interferometry, for instance in the $N$-band that is accessible with the VLTI/MIDI instrument and the Keck Nuller. This wavelength regime traces dust at temperatures down to $\sim 300 \mathrm{~K}$, probing also the potentially flared disk regions on tens of astronomical units (Fig. 3, left). MIR interferometry has been used to test SEDbased classification schemes about the disk flaring properties, such as the scheme by Meeus et al. (2001). In the scheme, Group I disks have a flat or rising mid-infrared SED, while Group II disks show a strong decline in the MIR. It has been proposed that Group I objects constitute flared disks, while Group II disks exhibit a flat or self-shadowed geometry, where the material near the dust sublimation rim casts a shadow on the outer disk. Interferometric studies confirm this idea: The MIDI survey by Leinert et al. (2004) showed that the MIRemitting size of seven HAeBe stars correlates with the mid-infrared (12-25 $\mu \mathrm{m})$ spectral slope, where Group I sources exhibit systematically larger sizes than Group II sources. More quantitative results were obtained using radiative transfer simulations on individual sources. For instance, di Folco et al. (2009) modelled MIDI data on the Group I source AB Aurigae and found a disk flaring index of $\beta=1.27 \pm 0.025$, which is consistent with the predicted value for a passive irradiated disk ( $\beta=9 / 7 \approx 1.29$, Chiang and Goldreich 1997). These authors also showed that the flaring index and the disk scale height at $100 \mathrm{au}\left(h_{100}=8 \pm 0.5 \mathrm{au}\right)$ were much better constrained by fitting the MIDI and SED data than by fitting the SED data alone. Keck interferometric observations on the Group II source MWC325, on the other hand, found a much lower flaring index $(0.85 \pm 0.13$, Ragland et al. 2012), consistent with a self-shadowed disk.

Recently, Maaskant et al. (2013) proposed an alternative scenario, where the SED-shape in Group I objects relates not necessarily to a flared disk geometry, but could indicate a gapped disk structure instead (similar to the pre-transitional disks that will be discussed in Sect. 4). It should be possible to test this scenario using 


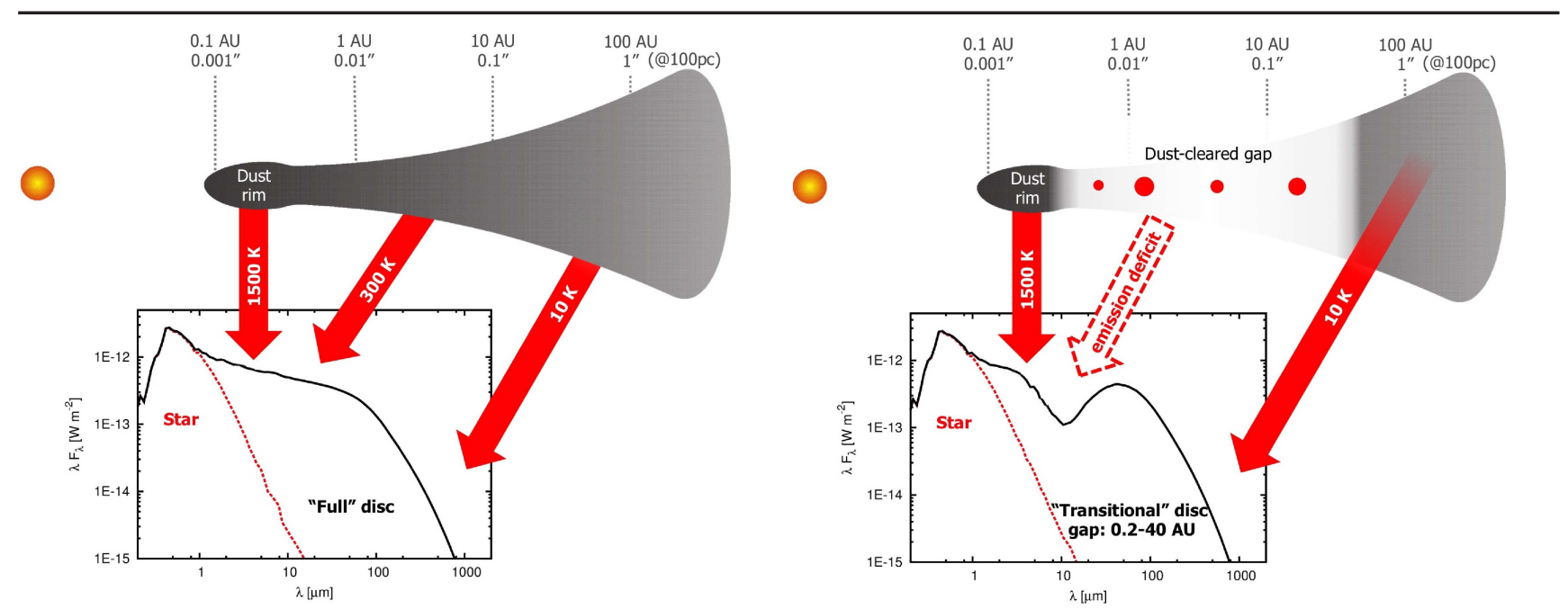

Fig. 3 Comparison of the SED and disk structure of classical "full" HAeBe disks (left) and pre-transitional disks (right). Following Wien's law, the shortest wavelength trace the hottest material closest to the star. For pre-transitional disks, a significant deficit in MIR emission is observed, which is interpreted as evidence for extended gaps in these systems, possibly revealing the presence of planets in these systems.

the improved $u v$-coverage and wavelength coverage $(L$, $M$, and $N$-band) that will be provided by the VLTI $2^{\text {nd }}$-generation instrument MATISSE. Further studies should also be able to reveal whether Group I and Group II sources form an evolutionary sequence. Based on the idea that Group I have a flared and Group II have a flat geometry, Dullemond and Dominik (2004) proposed that disks might starts out with a flaring shape (Group I disks) and then go through the process of grain growth, causing the optical depth of the disk to drop and the disk to become self-shadowed (Group II disks). In the scenario by Maaskant et al., on the other hand, Group I and II disks do not form an evolutionary sequence, but both evolve from the primordial flaring disks as common ancestor.

\section{Disk gaps and asymmetries}

As the disk evolves, different mechanisms will start to disperse the disk material through viscous spreading, mass-loss in winds, photoevaporation, and other mechanisms (Alexander et al. 2013). Another important mechanism might be dynamical clearing by newlyformed planets, which could result in extended disk gaps. The planets are also expected to trigger asymmetric structures, such as spiral waves or disk warps. A class of disk that might be affected by such planetrelated clearing processes are the transitional and pretransitional disks. These disks have a strong farinfrared excess, but a strongly reduced near-infrared or mid-infrared excess, which is often interpreted as evidence for the presence of holes or gaps in these objects (Fig. 3, right). Interferometry has started to make important contributions towards understanding the structure of these objects. Evidence for disk gaps has been detected in four pre-transitional disks, including V1247 Orionis (Kraus et al. 2013), HD100546 (Benisty et al. 2010b; Tatulli et al. 2011; Mulders et al. 2013; Panić et al. 2014), T Chamaeleontis (Olofsson et al. 2011, 2013), and SAO 206462 (Fedele et al. 2008; Carmona et al. 2014). These disks exhibit very narrow optically thick inner components near the dust sublimation radius (width $\sim 0.1 \mathrm{au}$ ), followed by a gap and an optically thick outer disk that extends from a few tens of astronomical units outwards. V1247 Orionis is exceptional in the sense that the gap region is filled with large amount of optically thin material, which lead to the proposal that this object might exhibit an earlier stage of disk clearing than the other pre-transitional disks that have been studies with this approach so far (Kraus et al. 2013). Even earlier stages of gap-opening might be observed in the classical Herbig Ae stars HD179218 (Fedele et al. 2008) and HD139614 (Matter et al. 2014), where the modelling of MIDI data suggests inhomogeneities in the intensity profile that might indicate either narrow disk gaps or shadowed regions behind the dust sublimation rim. By contrast, TW Hydrae seems to be in a particularly late stage of disk evolution, with a highly depleted inner in the inner 2.5 au and large dust grains that are settled towards the disk mid-plane (Eisner et al. 2006; Ratzka et al. 2007; Akeson et al. 2011; Arnold et al. 2012; Menu et al. 2014).

A particularly intriguing result has been the detection of non-zero phase signals in pre-transitional disks, indicating asymmetries in the brightness distribution. 
Given the poor $u v$-coverage, it is often not possible to reconstruct a model-independent image of the asymmetries, but geometric models are employed to reproduce the measured phase signals. For instance, the NIR aperture masking observations on LkCa 15 (Kraus and Ireland 2012) could be reproduced with a simple companion model, where the detected companion might even be in the planetary-mass regime. Observations on T Chamaeleontis have also been interpreted with a companion scenario (Huélamo et al. 2011), even though it has been proposed that the phase signals could also be reproduced with the emission from heated dust at the inner wall of the outer disk (Olofsson et al. 2013). Another interesting case is the pre-transitional disk of V1247 Orionis, where strong asymmetries were detected in multiple filters $\left(H+K^{\prime}+L^{\prime}\right.$ band) yet the amplitude and direction of the asymmetry changes between the different filters. This is not consistent with a singlecompanion interpretation but likely indicates complex density structures in the disk itself.

Recent studies also started to explore how the structure of the dust disk affects the physical conditions of the line-emitting gas. For instance, Carmona et al. (2014) presented gas+dust radiative transfer simulations of the disk of SAO 206462 and showed that the profile of the $\mathrm{CO} 4.7 \mu \mathrm{m}$ line depends strongly on the structure of the inner dust disk. Modelling the line profiles and NIR continuum structure simultaneously allowed them to derive the gas-to-dust ratio and the gas surface density for different regions in the disk.

\section{Dust mineralogy constraints}

The mid-infrared $N$-band contains also several important dust opacity features that can be accessed with spectrally dispersed mid-infrared interferometric instruments. First observations on young stars using this technique were presented by van Boekel et al. (2004) and targeted three Herbig stars with the MIDI instrument. Comparing the MIDI correlated flux spectra $F_{\text {corr }}$ with the total flux spectra $F$ (e.g. as measured with a conventional spectrograph) reveals some significant differences in the shape of the $10 \mu \mathrm{m}$ Silicate feature. The total flux spectra are representative of ISM-like dust, while the correlated flux spectra reveal also some spectral features that are indicative of crystalline dust. Given that the correlated flux is the product of the total flux with the visibility amplitude $\left(F_{\text {corr }}=F \cdot V\right)$, these differences can be interpreted with a spatial gradient in dust mineralogy, where the ISM-like dust is located on larger stellocentric radii and are therefore resolved out on the interferometric baseline. So far, this method has been applied only to a few HAeBe and T Tauri stars (Schegerer et al. 2009), but this is certainly a very promising topic for further studies.

\section{Gas kinematics constraints}

While the circumstellar dust provides most of the infrared continuum opacity, the mass of pre-mainsequence disks is dominated by circumstellar gas. Spectrally dispersed interferometric observations allow us to trace this gas content directly, for instance through observations in the $\mathrm{Br} \gamma 2.16 \mu \mathrm{m}$ or $\mathrm{H} \alpha 656 \mathrm{~nm}$ hydrogen recombination line, or in the $\mathrm{CO}$ bandheads at $2.3 \mu \mathrm{m}$. These lines are commonly observed towards HAeBe stars, but it is still unknown which processes in the disk are traced by these diagnostic lines.

Spectroscopic surveys showed that the equivalent width of the $\operatorname{Br} \gamma$ line correlates with the mass accretion rate, which has resulted in the idea that this line is a direct tracer of the hot gas that is transported onto the stellar surface through magnetospheric accretion columns. Observations with the VLTI/AMBER and Keck/V2-SPR instrument support this interpretation for T Tauri and very late-type Herbig Ae stars, where the visibilities measured within the line indicate that the line emission originates from just a few stellar radii from the star (Kraus et al. 2009a; Eisner et al. 2010, 2014). However, many high-luminosity sources show a significantly more extended line-emission region, which is not consistent with a magnetospheric accretion scenario. For instance, the $\mathrm{Br} \gamma$-line emission of the Herbig B[e] stars MWC297 (Malbet et al. 2007; Kraus et al. 2008b; Weigelt et al. 2011), MWC 158 (Ellerbroek et al. 2014), and V921 Scorpii (Kraus et al. 2012b) extends $\sim 1.6 \mathrm{au}, 3 \mathrm{au}$, and $6.7 \mathrm{au}$, respectively. Given this surprising finding that the $\mathrm{Br} \gamma$-line can trace both mass infall and outflow, it has been proposed that at least for some HAeBe stars, $\mathrm{Br} \gamma$ is not a primary tracer of accretion, but indirectly linked to the accretion rate, e.g. via accretion-driven mass loss (Kraus et al. 2008b).

Besides measuring the "integrated" size of the lineemitting region, some observations were also able to spectrally resolve the line and to study the geometry of the line-emitting region as function of velocity. Such gas kinematics studies can be conducted using AMBER's unique high spectral dispersion mode of $R=\lambda / \Delta \lambda=$ 12 000. For the Herbig B[e] stars V921 Scorpii (Kraus et al. 2012a) and MWC158 (Ellerbroek et al. 2014), it was found that the $\mathrm{Br} \gamma$-emitting gas is in Keplerian rotation in the inner disk. Observations on MWC297, on the other hand, had to invoked a more complex disk wind model (Weigelt et al. 2011). 
Also the $\mathrm{H} \alpha$ line has been associated both with accretion and outflow processes and spectroscopic observations are often not able to discern between these different mechansims. Visual-wavelength interferometry, for instance with the CHARA/VEGA instrument, can provide now fundamentally new constraints to distinguish between these models. So far, $\mathrm{H} \alpha$ spectrointerferometry have been reported for only two HAeBe stars, namely AB Aurigae (Rousselet-Perraut et al. 2010) and MWC158 (Ellerbroek et al. 2014). In both cases, the measurements indicate that the line-emitting region is more extended than what would be expected from a magnetosphere accretion scenario and the measurements appear more consistent with an X-wind or disk-wind geometry, although it should be noted that the measurement uncertainties are still very large in the current data sets.

Besides the hydrogen recombination lines, spectrointerferometric observations have also been conducted in the $2.3 \mu \mathrm{m}$ bandhead lines of carbon monoxide $(\mathrm{CO})$. Spectroscopic observations suggest that the CO line emission originates from interior to the dust sublimation radius, but outside the corotation radius of the central stars (Ilee et al. 2014). Observations on the Herbig Be star 51 Ophiuchi (Tatulli et al. 2008), the B[e] star HD 85567 (Wheelwright et al. 2013), and the lowermass objects AS 353A and V1331 Cyg (Eisner et al. 2014) confirm this hypothesis. With a spectral resolution $R \lesssim 2000$, the existing observations are not yet able to resolve the individual bandheads, but this could be achieved with future observations using AMBER's high spectral resolution mode.

\section{Multiplicity studies}

Detecting binaries and tracing their orbital motion is a classical application of optical long-baseline interferometry. Optical interferometric observations have lead to the discovery of previously unknown companions around HAeBe stars, including V921 Scorpii (Kraus et al. 2012b) and MWC300 (Wang et al. 2012), and provided orbit measurements of previously known binaries, such as MWC361 (Millan-Gabet et al. 2001; Monnier et al. 2006) and HD104237 (Garcia et al. 2013). With separations of a few astronomical units, the orbital period of these systems is relatively short (few years to a few tens of years), which makes it possible to trace the orbital motion of these systems on convenient timescales. Combining such astrometric orbits with radial velocity measurements can yield direct measurements of the system mass, providing important input for the calibration of pre-main-sequence evolutionary tracks.
For three systems, it has also been possible to obtain spectro-interferometric observations in spectral lines at different phases in the binary orbit and to investigate whether the companion might trigger enhanced massaccretion events close to periastron passage. Observations with the CHARA/VEGA instrument targeted the Herbig Be star MWC361 in the $\mathrm{H} \alpha$ line (Benisty et al. 2013) and found that the equivalent width as well as the spatial extend of the $\mathrm{H} \alpha$-line emitting region increased close to periastron passage. The authors propose that this might indicate the presence of a non-spherical wind that is enhanced at periastron. Observations on the Herbig Ae star HD 104237 targeted the $\operatorname{Br} \gamma$ line and found also here an equivalent width increase by factor $\sim 2$ near periastron (Garcia et al. 2013). The authors suggest a scenario, where a stellar wind is collimated by a circumbinary disk wind.

\section{Conclusions}

Optical interferometry has matured considerably over the last decade and HAeBe disk research is the field that might have benefitted the most from these advancements, both due to the favourable infrared brightness of these objects and their relatively large angular size. It is clear that the existing studies are still limited by the poor $u v$-coverage and the use of moderate baseline lengths, which restricts the possibilities for studying complex disk structures and for obtaining model-independent results. The upcoming generation of VLTI 4-telescope instruments and possible new CHARA 6-telescope beam combiner will mitigate these limiations and enable routine au-scale interferometric imaging, which will make optical interferometry also more accessible for non-experts. In the longer future, facilities with larger number of apertures could image the complex structures that are expected at different stages of protoplanetary disks evolution and planet formation (Monnier et al. 2014; Kraus et al. 2014), unleashing the full potential of this fascinating technique.

Acknowledgements I acknowledge support from an STFC Ernest Rutherford fellowship and the EU-funded Marie Curie CIG grant SH-06192. 


\section{References}

Akeson, R.L., Ciardi, D.R., van Belle, G.T., CreechEakman, M.J., Lada, E.A.: Astrophys. J. 543, 313 (2000). arXiv:astro-ph/0006025. doi:10.1086/317111

Akeson, R.L., Millan-Gabet, R., Ciardi, D.R., Boden, A.F., Sargent, A.I., Monnier, J.D., McAlister, H., ten Brummelaar, T., Sturmann, J., Sturmann, L., Turner, N.: Astrophys. J. 728, 96 (2011). doi:10.1088/0004$637 \mathrm{X} / 728 / 2 / 96$

Alexander, R., Pascucci, I., Andrews, S., Armitage, P., Cieza, L.: ArXiv e-prints (2013). 1311.1819

Arnold, T.J., Eisner, J.A., Monnier, J.D., Tuthill, P.: Astrophys. J. 750, 119 (2012). 1204.0550. doi:10.1088/0004$637 \mathrm{X} / 750 / 2 / 119$

Bans, A., Königl, A.: Astrophys. J. 758, 100 (2012). 1207.1508. doi:10.1088/0004-637X/758/2/100

Benisty, M., Natta, A., Isella, A., Berger, J.-P., Massi, F., Le Bouquin, J.-B., Mérand, A., Duvert, G., Kraus, S., Malbet, F., Olofsson, J., Robbe-Dubois, S., Testi, L., Vannier, M., Weigelt, G.: Astron. Astrophys. 511, 74 (2010a). 0911.4363. doi:10.1051/0004-6361/200912898

Benisty, M., Tatulli, E., Ménard, F., Swain, M.R.: Astron. Astrophys. 511, 75 (2010b). 1001.2491. doi:10.1051/0004$6361 / 200913590$

Benisty, M., Perraut, K., Mourard, D., Stee, P., Lima, G.H.R.A., Le Bouquin, J.B., Borges Fernandes, M., Chesneau, O., Nardetto, N., Tallon-Bosc, I., McAlister, H., Ten Brummelaar, T., Ridgway, S., Sturmann, J., Sturmann, L., Turner, N., Farrington, C., Goldfinger, P.J.: Astron. Astrophys. 555, 113 (2013). 1306.0390. doi:10.1051/0004-6361/201219893

Carmona, A., Pinte, C., Thi, W.F., Benisty, M., Ménard, F., Grady, C., Kamp, I., Woitke, P., Olofsson, J., Roberge, A., Brittain, S., Duchêne, G., Meeus, G., Martin-Zaïdi, C., Dent, B., Le Bouquin, J.B., Berger, J.P.: Astron. Astrophys. 567, 51 (2014). 1403.6193. doi:10.1051/0004-6361/201322534

Chiang, E.I., Goldreich, P.: Astrophys. J. 490, 368 (1997). astro-ph/9706042

di Folco, E., Dutrey, A., Chesneau, O., Wolf, S., Schegerer, A., Leinert, C., Lopez, B.: Astron. Astrophys. 500, 1065 (2009). doi:10.1051/0004-6361/200809902

Dullemond, C.P., Dominik, C.: Astron. Astrophys. 417, 159 (2004). astro-ph/0401495. doi:10.1051/0004-6361:20031768

Dullemond, C.P., Dominik, C., Natta, A.: Astrophys. J. 560, 957 (2001). arXiv:astro-ph/0106470

Eisner, J.A., Chiang, E.I., Hillenbrand, L.A.: Astrophys. J. Lett. 637, 133 (2006). astro-ph/0601034. doi:10.1086/500689

Eisner, J.A., Hillenbrand, L.A., Stone, J.M.: Mon. Not. R. Astron. Soc. 443, 1916 (2014). arXiv:astro-ph/1406.6402. doi:10.1093/mnras/stu1281

Eisner, J.A., Lane, B.F., Hillenbrand, L.A., Akeson, R.L., Sargent, A.I.: Astrophys. J. 613, 1049 (2004). arXiv:astroph/0406356. doi:10.1086/423314

Eisner, J.A., Chiang, E.I., Lane, B.F., Akeson, R.L.: Astrophys. J. 657, 347 (2007). arXiv:astro-ph/0611447. doi:10.1086/510833
Eisner, J.A., Monnier, J.D., Woillez, J., Akeson, R.L., Millan-Gabet, R., Graham, J.R., Hillenbrand, L.A., Pott, J., Ragland, S., Wizinowich, P.: Astrophys. J. 718, 774 (2010). 1006.1651. doi:10.1088/0004-637X/718/2/774

Ellerbroek, L.E., Benisty, M., Kraus, S., Perraut, K., Kluska, J., le Bouquin, J.B., Borges Fernandes, M., Domiciano de Souza, A., Maaskant, K.M., Kaper, L., Tramper, F., Mourard, D., Tallon-Bosc, I., ten Brummelaar, T., Sitko, M.L., Lynch, D.K., Russell, R.W.: ArXiv e-prints (2014). 1409.7394

Fedele, D., van den Ancker, M.E., Acke, B., van der Plas, G., van Boekel, R., Wittkowski, M., Henning, T., Bouwman, J., Meeus, G., Rafanelli, P.: Astron. Astrophys. 491, 809 (2008). 0809.3947. doi:10.1051/0004-6361:200810126

Garcia, P.J.V., Benisty, M., Dougados, C., Bacciotti, F., Clausse, J.-M., Massi, F., Mérand, A., Petrov, R., Weigelt, G.: Mon. Not. R. Astron. Soc. 430, 1839 (2013). 1301.0276. doi:10.1093/mnras/stt005

Hillenbrand, L.A., Strom, S.E., Vrba, F.J., Keene, J.: Astrophys. J. 397, 613 (1992). doi:10.1086/171819

Huélamo, N., Lacour, S., Tuthill, P., Ireland, M., Kraus, A., Chauvin, G.: Astron. Astrophys. 528, 7 (2011). 1102.4982. doi:10.1051/0004-6361/201016395

Ilee, J.D., Fairlamb, J., Oudmaijer, R.D., Mendigutia, I., van den Ancker, M., Kraus, S., Wheelwright, H.E.: ArXiv e-prints (2014). 1409.4897

Isella, A., Natta, A.: Astron. Astrophys. 438, 899 (2005). arXiv:astro-ph/0503635. doi:10.1051/0004-6361:20052773

Kluska, J., Malbet, F., Berger, J.-P., Baron, F., Lazareff, B., Le Bouquin, J.-B., Monnier, J.D., Soulez, F., Thiébaut, E.: Astron. Astrophys. 564, 80 (2014). 1403.3343. doi:10.1051/0004-6361/201322926

Kraus, A.L., Ireland, M.J.: Astrophys. J. 745, 5 (2012). 1110.3808. doi:10.1088/0004-637X/745/1/5

Kraus, S., Preibisch, T., Ohnaka, K.: Astrophys. J. 676, 490 (2008a). arXiv:0711.4988. doi:10.1086/527427

Kraus, S., Hofmann, K.-H., Benisty, M., Berger, J.-P., Chesneau, O., Isella, A., Malbet, F., Meilland, A., Nardetto, N., Natta, A., Preibisch, T., Schertl, D., Smith, M., Stee, P., Tatulli, E., Testi, L., Weigelt, G.: Astron. Astrophys. 489, 1157 (2008b). 0807.1119. doi:10.1051/0004-6361:200809946

Kraus, S., Hofmann, K., Malbet, F., Meilland, A., Natta, A., Schertl, D., Stee, P., Weigelt, G.: Astron. Astrophys. 508, 787 (2009a). 0911.3653. doi:10.1051/0004$6361 / 200912990$

Kraus, S., Weigelt, G., Balega, Y.Y., Docobo, J.A., Hofmann, K.-H., Preibisch, T., Schertl, D., Tamazian, V.S., Driebe, T., Ohnaka, K., Petrov, R., Schöller, M., Smith, M.: Astron. Astrophys. 497, 195 (2009b). 0902.0365. doi:10.1051/0004-6361/200810368

Kraus, S., Hofmann, K., Menten, K.M., Schertl, D., Weigelt, G., Wyrowski, F., Meilland, A., Perraut, K., Petrov, R., Robbe-Dubois, S., Schilke, P., Testi, L.: Nature 466, 339 (2010). 1007.5062. doi:10.1038/nature09174

Kraus, S., Calvet, N., Hartmann, L., Hofmann, K.H., Kreplin, A., Monnier, J.D., Weigelt, G.: Astrophys. J. 752, 11 (2012a). 1204.1969. doi:10.1088/0004$637 X / 752 / 1 / 11$

Kraus, S., Calvet, N., Hartmann, L., Hofmann, K.-H., Kreplin, A., Monnier, J.D., Weigelt, G.: ArXiv e-prints (2012b). 1100.0000 
Kraus, S., Ireland, M.J., Sitko, M.L., Monnier, J.D., Calvet, N., Espaillat, C., Grady, C.A., Harries, T.J., Hönig, S.F., Russell, R.W., Swearingen, J.R., Werren, C., Wilner, D.J.: Astrophys. J. 768, 80 (2013). 1304.2768. doi:10.1088/0004-637X/768/1/80

Kraus, S., Monnier, J., Harries, T., Dong, R., Bate, M., Whitney, B., Zhu, Z., Buscher, D., Berger, J.-P., Haniff, C., Ireland, M., Labadie, L., Lacour, S., Petrov, R., Ridgway, S., Surdej, J., ten Brummelaar, T., Tuthill, P., van Belle, G.: ArXiv e-prints (2014). 1407.7033

Kreplin, A., Weigelt, G., Kraus, S., Grinin, V., Hofmann, K.-H., Kishimoto, M., Schertl, D., Tambovtseva, L., Clausse, J.-M., Massi, F., Perraut, K., Stee, P.: Astron. Astrophys. 551, 21 (2013). doi:10.1051/0004$6361 / 201220806$

Leinert, C., Haas, M., Ábrahám, P., Richichi, A.: Astron. Astrophys. 375, 927 (2001). doi:10.1051/00046361:20010904

Leinert, C., van Boekel, R., Waters, L.B.F.M., Chesneau, O., Malbet, F., Köhler, R., Jaffe, W., Ratzka, T., Dutrey, A., Preibisch, T., Graser, U., Bakker, E., Chagnon, G., Cotton, W.D., Dominik, C., Dullemond, C.P., Glazenborg-Kluttig, A.W., Glindemann, A., Henning, T., Hofmann, K.-H., de Jong, J., Lenzen, R., Ligori, S., Lopez, B., Meisner, J., Morel, S., Paresce, F., Pel, J.W., Percheron, I., Perrin, G., Przygodda, F., Richichi, A., Schöller, M., Schuller, P., Stecklum, B., van den Ancker, M.E., von der Lühe, O., Weigelt, G.: Astron. Astrophys. 423, 537 (2004). doi:10.1051/0004-6361:20047178

Maaskant, K.M., Honda, M., Waters, L.B.F.M., Tielens, A.G.G.M., Dominik, C., Min, M., Verhoeff, A., Meeus, G., van den Ancker, M.E.: Astron. Astrophys. 555, 64 (2013). 1305.3138. doi:10.1051/0004-6361/201321300

Malbet, F., Benisty, M., de Wit, W.-J., Kraus, S., Meilland, A., Millour, F., Tatulli, E., Berger, J.-P., Chesneau, O., Hofmann, K.-H., Isella, A., Natta, A., Petrov, R.G., Preibisch, T., Stee, P., Testi, L., Weigelt, G., Antonelli, P., Beckmann, U., Bresson, Y., Chelli, A., Dugué, M., Duvert, G., Gennari, S., Glück, L., Kern, P., Lagarde, S., Le Coarer, E., Lisi, F., Perraut, K., Puget, P., Rantakyrö, F., Robbe-Dubois, S., Roussel, A., Zins, G., Accardo, M., Acke, B., Agabi, K., Altariba, E., Arezki, B., Aristidi, E., Baffa, C., Behrend, J., Blöcker, T., Bonhomme, S., Busoni, S., Cassaing, F., Clausse, J.-M., Colin, J., Connot, C., Delboulbé, A., Domiciano de Souza, A., Driebe, T., Feautrier, P., Ferruzzi, D., Forveille, T., Fossat, E., Foy, R., Fraix-Burnet, D., Gallardo, A., Giani, E., Gil, C., Glentzlin, A., Heiden, M., Heininger, M., Hernandez Utrera, O., Kamm, D., Kiekebusch, M., Le Contel, D., Le Contel, J.-M., Lesourd, T., Lopez, B., Lopez, M., Magnard, Y., Marconi, A., Mars, G., Martinot-Lagarde, G., Mathias, P., Mège, P., Monin, J.-L., Mouillet, D., Mourard, D., Nussbaum, E., Ohnaka, K., Pacheco, J., Perrier, C., Rabbia, Y., Rebattu, S., Reynaud, F., Richichi, A., Robini, A., Sacchettini, M., Schertl, D., Schöller, M., Solscheid, W., Spang, A., Stefanini, P., Tallon, M., Tallon-Bosc, I., Tasso, D., Vakili, F., von der Lühe, O., Valtier, J.-C., Vannier, M., Ventura, N.: Astron. Astrophys. 464, 43 (2007). doi:10.1051/00046361:20053924
Matter, A., Labadie, L., Kreplin, A., Lopez, B., Wolf, S., Weigelt, G., Ertel, S., Pott, J.-U., Danchi, W.C.: Astron. Astrophys. 561, 26 (2014). 1311.5131. doi:10.1051/0004$6361 / 201322042$

Meeus, G., Waters, L.B.F.M., Bouwman, J., van den Ancker, M.E., Waelkens, C., Malfait, K.: Astron. Astrophys. 365, 476 (2001). astro-ph/0012295. doi:10.1051/00046361:20000144

Menu, J., van Boekel, R., Henning, T., Chandler, C.J., Linz, H., Benisty, M., Lacour, S., Min, M., Waelkens, C., Andrews, S.M., Calvet, N., Carpenter, J.M., Corder, S.A., Deller, A.T., Greaves, J.S., Harris, R.J., Isella, A., Kwon, W., Lazio, J., Le Bouquin, J.-B., Ménard, F., Mundy, L.G., Pérez, L.M., Ricci, L., Sargent, A.I., Storm, S., Testi, L., Wilner, D.J.: Astron. Astrophys. 564, 93 (2014). 1402.6597. doi:10.1051/0004-6361/201322961

Millan-Gabet, R., Schloerb, F.P., Traub, W.A.: Astrophys. J. 546, 358 (2001). astro-ph/0008072. doi:10.1086/318239

Millan-Gabet, R., Schloerb, F.P., Traub, W.A., Malbet, F., Berger, J.P., Bregman, J.D.: Astrophys. J. Lett. 513, 131 (1999). doi:10.1086/311926

Millan-Gabet, R., Monnier, J.D., Berger, J.-P., Traub, W.A., Schloerb, F.P., Pedretti, E., Benisty, M., Carleton, N.P., Haguenauer, P., Kern, P., Labeye, P., Lacasse, M.G., Malbet, F., Perraut, K., Pearlman, M., Thureau, N.: Astrophys. J. Lett. 645, 77 (2006). arXiv:astroph/0606059. doi:10.1086/506153

Monnier, J.D., Millan-Gabet, R.: Astrophys. J. 579, 694 (2002). arXiv:astro-ph/0207292. doi:10.1086/342917

Monnier, J.D., Millan-Gabet, R., Billmeier, R., Akeson, R.L., Wallace, D., Berger, J.-P., Calvet, N., D'Alessio, P., Danchi, W.C., Hartmann, L., Hillenbrand, L.A., Kuchner, M., Rajagopal, J., Traub, W.A., Tuthill, P.G., Boden, A., Booth, A., Colavita, M., Gathright, J., Hrynevych, M., Le Mignant, D., Ligon, R., Neyman, C., Swain, M., Thompson, R., Vasisht, G., Wizinowich, P., Beichman, C., Beletic, J., Creech-Eakman, M., Koresko, C., Sargent, A., Shao, M., van Belle, G.: Astrophys. J. 624, 832 (2005). arXiv:astro-ph/0502252. doi:10.1086/429266

Monnier, J.D., Berger, J.-P., Millan-Gabet, R., Traub, W.A., Schloerb, F.P., Pedretti, E., Benisty, M., Carleton, N.P., Haguenauer, P., Kern, P., Labeye, P., Lacasse, M.G., Malbet, F., Perraut, K., Pearlman, M., Zhao, M.: Astrophys. J. 647, 444 (2006). astro-ph/0606052. doi:10.1086/505340

Monnier, J.D., Kraus, S., Buscher, D., Berger, J.-P., Haniff, C., Ireland, M., Labadie, L., Lacour, S., Le Coroller, H., Petrov, R.G., Pott, J.-U., Ridgway, S., Surdej, J., ten Brummelaar, T., Tuthill, P., van Belle, G.: ArXiv e-prints (2014). 1407.7032

Mulders, G.D., Paardekooper, S.-J., Panić, O., Dominik, C., van Boekel, R., Ratzka, T.: Astron. Astrophys. 557, 68 (2013). 1306.4264. doi:10.1051/0004-6361/201220930

Muzerolle, J., D'Alessio, P., Calvet, N., Hartmann, L.: Astrophys. J. 617, 406 (2004). astro-ph/0409008. doi:10.1086/425260

Natta, A., Prusti, T., Neri, R., Wooden, D., Grinin, V.P., Mannings, V.: Astron. Astrophys. 371, 186 (2001). doi:10.1051/0004-6361:20010334 
Olofsson, J., Benisty, M., Augereau, J.-C., Pinte, C., Ménard, F., Tatulli, E., Berger, J.-P., Malbet, F., Merín, B., van Dishoeck, E.F., Lacour, S., Pontoppidan, K.M., Monin, J.-L., Brown, J.M., Blake, G.A.: Astron. Astrophys. 528, 6 (2011). 1102.4976. doi:10.1051/0004$6361 / 201016074$

Olofsson, J., Benisty, M., Le Bouquin, J.-B., Berger, J.-P., Lacour, S., Ménard, F., Henning, T., Crida, A., Burtscher, L., Meeus, G., Ratzka, T., Pinte, C., Augereau, J.-C., Malbet, F., Lazareff, B., Traub, W.: Astron. Astrophys. 552, 4 (2013). 1302.2622. doi:10.1051/0004 6361/201220675

Panić, O., Ratzka, T., Mulders, G.D., Dominik, C., van Boekel, R., Henning, T., Jaffe, W., Min, M.: Astron. Astrophys. 562, 101 (2014). 1203.6265. doi:10.1051/0004$6361 / 201219223$

Pinte, C., Ménard, F., Berger, J.P., Benisty, M., Malbet, F.: Astrophys. J. Lett. 673, 63 (2008). arXiv:0712.0012. doi:10.1086/527378

Ragland, S., Ohnaka, K., Hillenbrand, L., Ridgway, S.T., Colavita, M.M., Akeson, R.L., Cotton, W., Danchi, W.C., Hrynevich, M., Millan-Gabet, R., Traub, W.A.: Astrophys. J. 746, 126 (2012). 1111.1813. doi:10.1088/0004$637 \mathrm{X} / 746 / 2 / 126$

Ratzka, T., Leinert, C., Henning, T., Bouwman, J., Dullemond, C.P., Jaffe, W.: Astron. Astrophys. 471, 173 (2007). 0707.0193. doi:10.1051/0004-6361:20077357

Renard, S., Malbet, F., Benisty, M., Thiébaut, E., Berger, J.-P.: Astron. Astrophys. 519, 26 (2010). 1007.2930. doi:10.1051/0004-6361/201014910

Rousselet-Perraut, K., Benisty, M., Mourard, D., Rajabi, S., Bacciotti, F., Bério, P., Bonneau, D., Chesneau, O., Clausse, J.M., Delaa, O., Marcotto, A., Roussel, A., Spang, A., Stee, P., Tallon-Bosc, I., McAlister, H., ten Brummelaar, T., Sturmann, J., Sturmann, L., Turner, N., Farrington, C., Goldfinger, P.J.: Astron. Astrophys. 516, 1 (2010). doi:10.1051/0004-6361/201014720

Schegerer, A.A., Wolf, S., Hummel, C.A., Quanz, S.P., Richichi, A.: Astron. Astrophys. 502, 367 (2009). 0905.0565. doi:10.1051/0004-6361/200810782

Tannirkulam, A., Monnier, J.D., Millan-Gabet, R., Harries, T.J., Pedretti, E., ten Brummelaar, T.A., McAlister, H., Turner, N., Sturmann, J., Sturmann, L.: Astrophys. J. Lett. 677, 51 (2008). arXiv:0803.1484. doi:10.1086/587873

Tatulli, E., Malbet, F., Ménard, F., Gil, C., Testi, L., Natta, A., Kraus, S., Stee, P., Robbe-Dubois, S.: Astron. Astrophys. 489, 1151 (2008). 0806.4937. doi:10.1051/00046361:200809627

Tatulli, E., Benisty, M., Ménard, F., Varnière, P., MartinZaïdi, C., Thi, W.-F., Pinte, C., Massi, F., Weigelt, G., Hofmann, K.-H., Petrov, R.G.: Astron. Astrophys. 531, 1 (2011). 1104.0905. doi:10.1051/0004-6361/201016165

Thamm, E., Steinacker, J., Henning, T.: Astron. Astrophys. 287, 493 (1994)

Turner, N.J., Benisty, M., Dullemond, C.P., Hirose, S.: Astrophys. J. 780, 42 (2014). 1309.0762. doi:10.1088/0004$637 \mathrm{X} / 780 / 1 / 42$ van Boekel, R., Min, M., Leinert, C., Waters, L.B.F.M., Richichi, A., Chesneau, O., Dominik, C., Jaffe, W., Dutrey, A., Graser, U., Henning, T., de Jong, J., Köhler, R., de Koter, A., Lopez, B., Malbet, F., Morel, S., Paresce, F., Perrin, G., Preibisch, T., Przygodda, F., Schöller, M., Wittkowski, M.: Nature 432, 479 (2004). doi: $10.1038 /$ nature03088

Wang, Y., Weigelt, G., Kreplin, A., Hofmann, K.-H., Kraus, S., Miroshnichenko, A.S., Schertl, D., Chelli, A., Domiciano de Souza, A., Massi, F., Robbe-Dubois, S.: Astron. Astrophys. 545, 10 (2012). 1208.5882. doi:10.1051/0004$6361 / 201219800$

Weigelt, G., Grinin, V.P., Groh, J.H., Hofmann, K.-H., Kraus, S., Miroshnichenko, A.S., Schertl, D., Tambovtseva, L.V., Benisty, M., Driebe, T., Lagarde, S., Malbet, F., Meilland, A., Petrov, R., Tatulli, E.: Astron. Astrophys. 527, 103 (2011). 1101.3695. doi:10.1051/0004$6361 / 201015676$

Wheelwright, H.E., Weigelt, G., Caratti o Garatti, A., Garcia Lopez, R.: Astron. Astrophys. 558, 116 (2013). 1308.6000. doi:10.1051/0004-6361/201322128 